\title{
Numerical simulation of fluid structure interaction, application to vibroacoustic problems
}

\author{
Ahlem Alia ${ }^{*, * *}$ — Jacques Charley ${ }^{*, * * *}$ \\ *Laboratoire de mécanique de Lille - LML \\ ** USTL, Cité Scientifique, F-59655 Villeneuve d'Ascq \\ ahlem.alia@ed.univ-lille1.fr \\ *** ENSAM, 8 Bd Louis XIV, F-59046 Lille cedex \\ jacques.charley@ensam.fr
}

ABSTRACT. In the present work, the transient response of a mechanical system is computed first by using an explicit finite element method. By applying the FFT, it's transformed into frequency response which allows to use BEM for computing the noise radiated at any point into space. BEM is checked first for an acoustic problem before using it for a vibroacoustic application. The numerical examples show the efficiency of the present method.

RÉSUMÉ. Dans ce travail, la réponse temporelle d'un système mécanique est calculée en utilisant la méthode des éléments finis explicite. En appliquant la FFT, cette réponse est transformée dans le domaine fréquentiel permettant ainsi le recours à la méthode des éléments finis de surface (BEM) pour le calcul du champ de pression rayonnée dans l'espace. La BEM est validée, dans un premier temps, pour un problème acoustique avant d'être utilisée pour une application en vibroacoustique.

KEYWORDS: acoustics, weak vibroacoustic coupling, BEM, FEM.

MOTS-CLÉS : acoustique, couplage vibroacoustique faible, BEM, FEM.

DOI:10.3166/REMN.16.437-450@ 2007 Lavoisier, Paris. Tous droits réservés 


\section{Introduction}

Fluid structure modelling can be done using either coupled or uncoupled approach. In the fully coupled systems, structure and fluid need to be modelled simultaneously. The most popular methods are those involving Finite Element (FE) formulations for both fluid and structure (Ohayon et al., 1998). However, direct resolution of the coupled system is time consuming. To overcome this problem, modal analysis constitutes an interesting alternative. In fact, only some structural modes in vacuum and some rigid cavity modes are involved into analysis which reduce significantly the discretized problem size. But, unfortunately, this leads to a non symmetric eigen-value problem. For this, Sandberg (1995) used some transformations allowing to put the eigen-value problem into a symmetric form. However, this method can lead to significant errors if the truncation of structural and acoustic modes is not convenient. Tournour et al., (2000), on his turn, derived a pseudo static correction in order to improve the convergence by taking into account the dropped modes without increasing the dimensions of the governing system of equations.

For many problems, it is not necessary to consider full fluid structure interaction. For instance, in the vehicle box case, many authors have reported fully coupling modelling by assuming uncoupled interaction. In this formulation, the structural response in vacuum is computed first. The obtained results are taken as boundary conditions for the acoustic part of the problem. Hence, the structural vibrations excite the fluid whereas the structure is never influenced by the acoustic waves propagating through the fluid. It's obviously that the uncoupled approach is more simple to be implemented than the coupled one, since two smaller models are to be computed one after the other (Marburg, 2002). However, it is limited for only heavy structures and light fluid (air acoustics). For example, in underwater acoustics, the vibration behaviour of the submerged structures is influenced by the surrounded water because of the important value of its normal acoustic impedance (Estorff, 2000). In this case, strong coupling must be taken into account.

In this paper, both Finite and Boundary Element Methods are employed to simulate, in low frequency range, noise radiated in acoustic and vibroacoustic problems. The transient response of mechanical system is computed first by using an explicit finite element scheme. The FFT allows its transformation into the frequency domain. In the other hand, a variational BE code based on the velocity information issue from the FFT results is used to compute the acoustic pressure at any point in space. This weak structural acoustic coupling is presented by assuming that the acoustic pressure does not affect the structural vibration (Marburg, 2002). 


\section{Structural analysis}

\subsection{Governing equations}

Let $\Omega_{\mathrm{s}}$ the bounded domain occupied by the structure and $\mathrm{n}$ the external normal to the boundary $\mathrm{S}_{\mathrm{s}} \cup \mathrm{S}_{\mathrm{s} 0}$ of $\Omega_{\mathrm{s}}$ (Figure 1). In the case of elastic, linear and isotropic structure without any initial stress or strain in absence of body forces, the displacement $u$ satisfies the following elasto-dynamic equation:

$$
\sigma_{i j, j}(u)-\rho \frac{\partial^{2} u_{i}}{\partial t^{2}}=0 \quad \text { in } \Omega_{s}
$$

where $u_{i}$ is the displacement in the $i^{\text {th }}$ direction, $\rho$ is the material density. In what follows, $\sigma_{\mathrm{ij}}(\mathrm{u})$ will represent the stress which depends upon the displacement. For a given displacement on $\mathrm{S}_{\mathrm{s} 0}$, we have the following boundary condition:

$$
u=u_{s} \quad \text { on } S_{s 0}
$$

For a prescribed surface force $f$ on $\mathrm{S}_{\mathrm{s}}$, we can write the corresponding boundary condition as follows:

$$
\sigma_{i j}(u) n_{j}=f_{i} \quad \text { on } S_{s}
$$

Finally, initial conditions can be written as:

$$
\left\{\begin{array}{l}
u(x, t=0)=0 \\
\frac{\partial u(x, t=0)}{\partial t}=0
\end{array} \text { in } \Omega_{s}\right.
$$

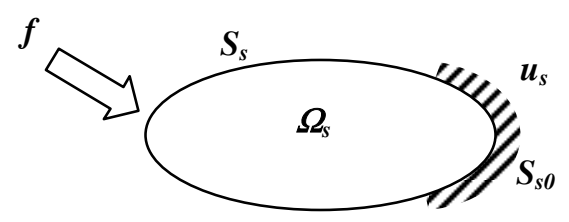

Figure 1. Structural domain and its boundaries

In the most applications, FE models were mainly investigated for simulation of structures. The detailed description of the used FE algorithm is not the goal of this paper. Generally, two methods can be used for analysing structural dynamics problems. The first one involves an implicit time integration scheme. In this method, 
the discretisation of the variational form involving any mechanical system response using the FEM reduces into the following linear system of equations:

$$
[K] u+[M] \frac{d^{2} u}{d t^{2}}=\{F\}
$$

where $t$ designs the time, $[\mathrm{M}]$ and $[\mathrm{K}]$ represent, respectively, the mass and the stiffness matrices of the structure and $\{\mathrm{F}\}$ is the mechanical load vector. To calculate the nodal displacement vector of the vibrating structure we need to solve a linear system. Note in this case, the scheme is unconditionally stable. The second method is based on an explicit scheme given by the following equation:

$$
M \ddot{u}_{n}=F_{n}^{e x t}-F_{n}^{i n t}
$$

Contrary to the implicit method, in the explicit one the CFL criterion limits the time step size to smaller value compared to that used by implicit scheme. In addition, this later, does not lead to linear system of equations. Since $M$ is lumped mass matrix, we use in this paper an explicit scheme to solve the structural part of the vibroacoustic problem.

Time derivation of the displacement vector leads to the velocity vector:

$$
\vec{v}(x, t)=\frac{\partial \vec{u}(x, t)}{\partial t}
$$

The normal component of the nodal velocity vector is extracted for all nodes located at the interface fluid structure as following:

$$
v_{n}(x, t)=\vec{v}(x, t) \cdot \vec{n}=\frac{\partial \vec{u}(x, t)}{\partial t} \cdot \vec{n}
$$

\subsection{Velocity in frequency domain}

The nodal velocities issues from the structural analysis are calculated in temporal domain. However, in order to use them as boundary conditions for the BEM, we need to transform them into frequency domain by using Fourier Transform:

$$
V(x, f)=\int_{-\infty}^{+\infty} v(x, t) e^{2 \pi i f t} d t
$$

However, $\mathrm{v}(\mathrm{x}, \mathrm{t})$ is a finite and sampled function with non uniform time sampling. In what follows, we need that this sampling rate be uniform. For this, we 
proceed by interpolation of $\mathrm{v}(\mathrm{x}, \mathrm{t})$ at a uniform $\Delta t$. Hence, we can approximate the integral in Equation [9] by a Discrete Fourier Transform (DFT):

$$
V\left(f_{n}\right)=\Delta t \sum_{k=0}^{N-l} v_{k} e^{2 \pi i k n / N}=\Delta t H_{n}
$$

where $\mathrm{N}$ is the number of input values.

On the other hand, inverse Fourier transform is given by:

$$
v_{k}=\frac{1}{N} \sum_{n=0}^{N-1} H_{n} e^{-2 \pi i k n / N}
$$

The total power in a function is the conserved whether we calculate it in frequency or time domain. This is known as Parseval's theorem:

$$
\sum_{k=0}^{N-l}\left|v_{k}\right|^{2}=\frac{1}{N} \sum_{n=0}^{N-l}\left|H_{n}\right|^{2}
$$

The FFT is an algorithm allowing to reduce the number of operations for computing the DFT. The most known one required $2^{\mathrm{n}}$ input values. In this paper, we use this simple algorithm to compute the FFT. So, the velocity interpolation is done for $2^{\mathrm{n}}$ uniform samples.

In many cases, it is difficult to obtain exactly a velocity signal formed by an integer number of cycles. The use of rectangular window can lead to important discontinuities since the generating data doesn't begin and end at the same level (non periodic signal). In this case, the FFT or DFT produces a spectrum with leakage (with parasite components in the spectrum). Windowing can force the data to begin and end at the same or nearly the same value. This can be accomplished by multiplying the sampled wave form data set by an appropriate windowing function. This prevents discontinuity at the window edge and consequently, helps to reduce leakage.

However when a window is used, it leads not only in a reducing of the leakage but also in a decreasing in the energetic content of the signal. It is possible to correct the DFT or FFT in order to compensate this energetic loss. This correction can be achieved by using Parseval's theorem (Equation [12]) which allows to evaluate a correction ratio due to the windowing.

\section{Acoustic analysis}

The BEM is used to evaluate the pressure response in the acoustic domain from the structure velocity results deduced from Equation [8]. Consider a boundary 
surface " $\mathrm{S}$ " enclosing a volume " $\Omega_{\mathrm{f}}$ " filled and surrounded by an ideal and homogeneous fluid medium (Figure 2).

\subsection{Governing equations}

For an harmonic disturbance of frequency "f" without any source or loss mechanisms, the integral form of the Helmholtz equation:

$$
\Delta p+k^{2} p=0
$$

can be easily deduced using Green's theorem. It allows the calculation of sound pressure at any point of the acoustic domain. It's given by this equation (Wu, 2000):

$$
C(r) p(r)=\int_{S_{y}}\left(G\left(r, r_{y}\right) \frac{\partial p(r)}{\partial n_{y}}-p \frac{\partial G\left(r, r_{y}\right)}{\partial n_{y}}\right) d S_{y}
$$

where $k=\frac{\omega}{c}$ denotes the wave number, $\mathrm{c}$ is the sound velocity, $\omega=2 \pi f$ is the pulsation, $\mathrm{p}(\mathrm{r})$ is the pressure at any field point " $\mathrm{r}$ ", $G\left(r, r_{y}\right)=\frac{e^{-i k\left|r-r_{y}\right|}}{4 \pi\left|r-r_{y}\right|}$ is the Green's function, " $\mathrm{r}$ " " is the position vector of a source point located at acoustic domain boundary and $\mathrm{C}(\mathrm{r})$ is the jump term resulting from the treatment of the singular integral involving Green's function.

Equation [14] represents the integral equation of the Direct Boundary Element Method (DBEM) in which the primary variables are the acoustic pressure and the normal velocity. It can solve either interior or exterior acoustic problems (Figure 2). The Indirect Boundary Element Method (IBEM) defines the primary variables as the difference in the pressure $(\mu)$ and the difference in the normal gradient of the pressure $(\sigma)$ between the two sides of the boundary element model.
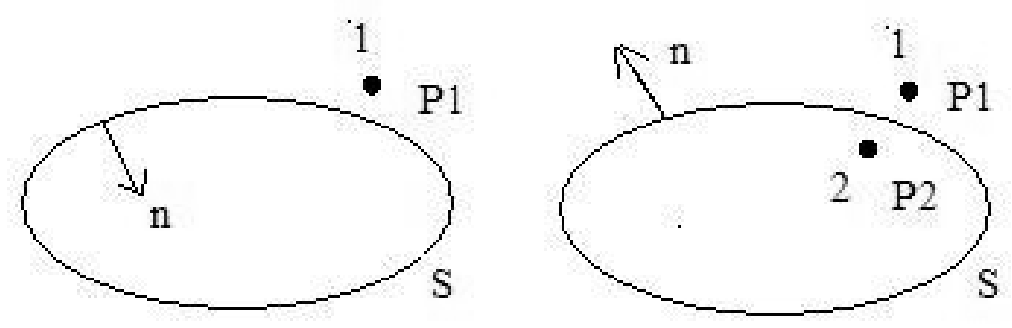

Figure 2. Acoustic domain and its boundary (left: DBEM, right: IBEM) 
For Neumann problem, in which only velocity is prescribed on the acoustic boundary, the following integral equation is obtained:

$$
p(r)=\int_{S_{y}} \frac{\partial G\left(r, r_{y}\right)}{\partial n_{y}} \mu\left(r_{y}\right) d S_{y}
$$

On the surface of the acoustic boundary, the pressure is related to the structural velocity by:

$$
\nabla p=-i \omega \rho v
$$

Hence, Equation [15] can be written as following:

$$
-i \omega \rho V_{n}\left(r_{y}\right)=\int_{S_{y}} \frac{\partial^{2} G\left(r, r_{y}\right)}{\partial n_{x} \partial n_{y}} \mu\left(r_{y}\right) d S_{y}
$$

\subsection{Variational Indirect BEM (VIBEM)}

By using the variational method, the last equation can be rewritten as:

$$
-i \omega \rho \int_{S_{x}} V_{n} \mu\left(r_{x}\right) d S_{x}=\int_{S_{x}} \int_{S_{y}} \frac{\partial^{2} G\left(r, r_{y}\right)}{\partial n_{x} \partial n_{y}} \mu\left(r_{x}\right) \mu\left(r_{y}\right) d S_{y} d S_{x}
$$

where $\mu\left(r_{x}\right)$ represents the test function of the variational method. The solution of the problem can be obtained by minimising the following functional $\mathrm{F}$ of the Variational Indirect Boundary Element Method (VIBEM):

$$
F(\mu)=2 \int_{S_{x}} j \rho \omega \mu\left(r_{x}\right) V_{n}\left(r_{x}\right) d S_{x}+\int_{S_{x}}\left(\mu\left(r_{x}\right) \int_{S_{y}}\left(\frac{\partial^{2} G\left(r_{x}, r_{y}\right)}{\partial n_{x} \partial n_{y}} \mu\left(r_{y}\right)\right) d S_{y}\right) d S_{x}
$$

The solution of Equation [17] can be obtained by dividing the surface into quadrilateral and/or triangular boundary elements. Hence, the discretized form of the functional $\mathrm{F}$ (see Equation [19]) noted $\mathrm{F}^{\mathrm{h}}$ may then be written as:

$$
F^{h}=\sum_{i} \sum_{j} \mu_{i} a_{i j} \mu_{j}-2 \sum_{i} \mu_{i} b_{i}
$$

or in matrix form:

$$
F^{h}=\mu^{T} A \mu-2 \mu^{T} B
$$


$\mathrm{F}^{\mathrm{h}}$ is a bilinear function of the unknown nodal potentials. Imposing stationary condition on $\mathrm{F}^{\mathrm{h}}$ with respect to unknown primary variables $\mu$, leads to the following system of equations:

$$
\frac{\partial F^{h}}{\partial \mu}=0 \Rightarrow A \mu=B
$$

where the global matrix $A$ is resulting from assembling the elementary matrices $a_{i j}$ and $\mathrm{B}$ results from assembling the elementary vectors $\mathrm{b}_{\mathrm{i}}$.

\subsection{Linear system solver}

The VIBEM gives rise to a linear system of equation in which the matrix A $(\mathrm{N} \times \mathrm{N})$ is complex, fully populated and symmetric. It can be solved directly or iteratively. Direct solvers are in common use but they become inefficient when applied to large problems. In last years, iterative solvers for BEM have been investigated by many researchers (Marburg et al., 2003). These solvers refer to a wide range of techniques that use successive approximations to obtain more accurate solutions to a linear system at each step. They are based on the idea of sequences of orthogonal vectors.

The Generalised Minimal Residual method (GMRES) is known as a very robust iterative method to solve non symmetric systems. To solve a linear problem $A x=b$, this method begins with an initial $x_{0}$ and at the $\mathrm{k}^{\text {th }}$ step, determines an iterate numerical results $x_{k}$ through a correction. A strong attraction of this method is that implementations only require matrix-vector products. In GMRES, each iterate $x_{k}$ is chosen to minimise the residual norm $\left\|b-A x_{k}\right\|_{2}$ over all corrections.

The major drawback of GMRES is that the amount of work and storage required per iteration rises linearly with the iteration count. It convergence is no more than $n$ steps which is of no practical value when $n$ is large. The usual way to overcome this storage limitation is by restarting the iterations. After a chosen number $\mathrm{m}$ of iterations, the accumulated data are cleared and the intermediate results are used as initial data for the next $\mathrm{m}$ iterations (Barrett, 1994). However, reduction of storage requirements (too small $\mathrm{m}$ ) will considerably slow convergence of the solver. Hence, a trade-off must be taken into account in order to achieve best convergence with the available storage (Marburg et al., 2003). 


\section{Numerical results}

\subsection{Application to automotive industry}

The geometry of real vibrating systems are often very complex. One efficient way to save time and memory is to approximate them by simpler geometry without loss of the most essential features of the vibratory and the acoustic problems. Figure 3 shows a simplified model of a vehicle compartment of $(1.4 \times 0.5 \times 0.6) \mathrm{m}^{3}$ filled by air $\left(\rho=1.23 \mathrm{Kg} / \mathrm{m}^{3}, c=340 \mathrm{~m} / \mathrm{s}\right)$. It is assumed that only the bottom of the car was vibrating as a rigid piston with an harmonic amplitude z-velocity of $0.007 \mathrm{~m} / \mathrm{s}$ whereas all other walls are rigid with zero particle velocity. We are interesting by computing the pressure at a point (see Figure 3 ) located at (482.923, 300., 312.5)mm inside the car by using FE and BE methods. The BE model is constituted by 1264 boundary elements and the FE one contains 2688 finite elements.
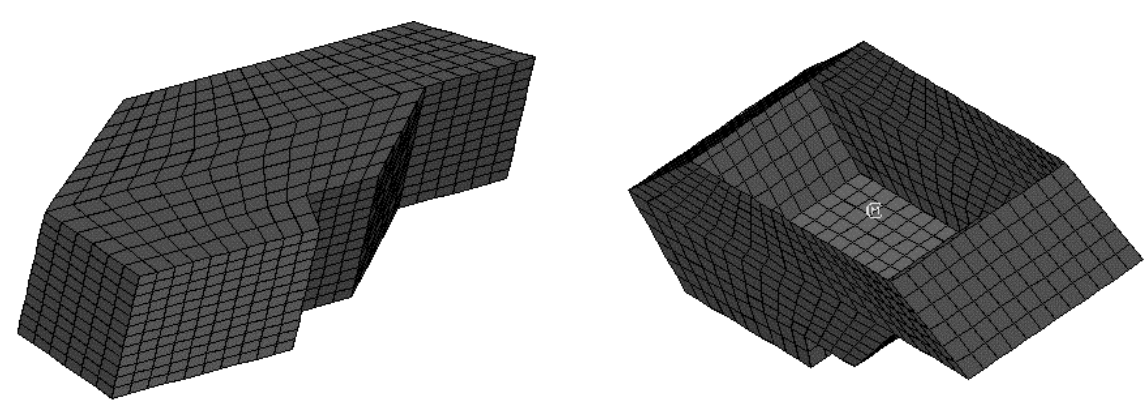

Figure 3. FE, BE models of the compartment

Simulations have been done for $10-500 \mathrm{~Hz}$ frequency range with a step of $1 \mathrm{~Hz}$. The pressure magnitude (in $\mathrm{dB}$ ) versus frequency $(\mathrm{Hz})$ is represented in Figure 4. It can be seen that the car presents a resonant behaviour at the compartment resonance which are calculated using FEM and are listed in Table 1. Figure 4 shows good agreement between FEM and BEM especially in low frequency range. In fact, for high frequencies, we have to use fine mesh to keep a certain accuracy.

For this problem, the FEM which is characteristically sparse, takes only $9 \mathrm{mn}$ to run on $2 \mathrm{GHz}$ CPU machine. However, the BEM takes $7 \mathrm{~h} 21 \mathrm{mn} 20$ s on the same machine when direct solver is applied by using 4 Gauss integration points. This CPU time is reduced to $3 \mathrm{~h} 30 \mathrm{mn} 31 \mathrm{~s}$ when GMRES is applied with a tolerance of $10^{-5}$ and maximum number of iterations $m=100$. Note that the use of 16 Gauss integration points doesn't lead to extra difference in the results but it increases dramatically the CPU time (about $58 \mathrm{~h}$ when direct solver is used). From Figure 5, we can deduce that good results can be curried out by using GMRES solver and considering 4 Gauss 
points. In what follows, we use GMRES solver to solve the linear system of equations. The BEM integrals are evaluated for 4 gauss integration points.

Table 1. Car resonance modes calculated by FEM

\begin{tabular}{|c|c|}
\hline Mode \# & Frequency $(\mathrm{Hz})$ \\
\hline 1 & 162.21 \\
\hline 2 & 284.14 \\
\hline 3 & 286.72 \\
\hline 4 & 327.18 \\
\hline 5 & 379.21 \\
\hline 6 & 401.56 \\
\hline 7 & 403.66 \\
\hline 8 & 473.85 \\
\hline 9 & 491.92 \\
\hline 10 & 506.77 \\
\hline
\end{tabular}

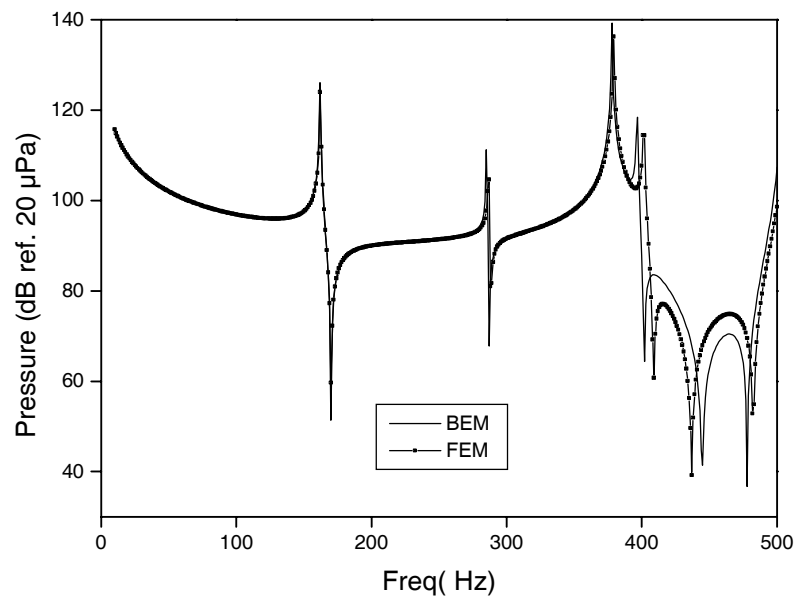

Figure 4. Variation of the acoustic pressure versus frequency 


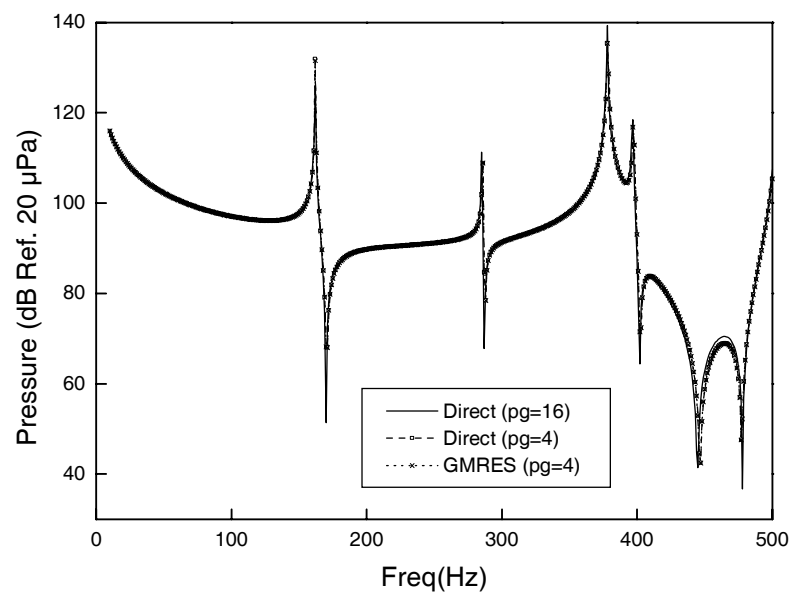

Figure 5. Acoustic pressure using BEM: comparison between direct and GMRES solver for 4 and 16 gauss integration points

\subsection{Elastic plate radiation}

In this second application, we are interesting by the calculation of the acoustic radiation of an elastic plate $(0.6 \times 0.9 \times 0.001) \mathrm{m}^{3}$ with the following mechanical properties: Young modulus $\mathrm{E}=210 \mathrm{GPa}, \rho_{\mathrm{s}}=7800 \mathrm{~kg} / \mathrm{m}^{3}$ and Poisson ratio $\mathrm{v}=0.3$. It's excited by a punctual unit Dirac force at a point located at $(0.33,0.45,0.) \mathrm{m}$. This plate is surrounded by air $\left(\rho=1.21 \mathrm{Kg} / \mathrm{m}^{3}, c=340 \mathrm{~m} / \mathrm{s}\right)$ and the acoustic pressure is to be calculated at a point located at $1 \mathrm{~m}$ from the plate (see Figure 6). The plate model is constituted from 600 boundary elements.

The mechanical velocity response of the excited structure is calculated for a duration of $1.023 \mathrm{~s}$ with a time step of $0.001 \mathrm{~s}$. Hence, the number of FFT input is equal to 1024 . In order to minimise the leackage we have used the window shown in Figure 7. Parseval's theorem is applied to correct the windowing effect.

In Figure 8, we have represented the acoustic response at the field point represented in Figure 6. In pure external radiation problems, the acoustic response is smooth and regular. However, when the acoustic problem involves elastic surfaces, strong peaks appear in the acoustic response due to the structure modes. In fact, pressure presents many peaks corresponding to the resonance frequencies of the elastic plate. 


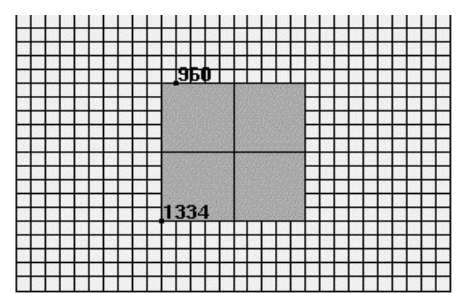

Field Poin
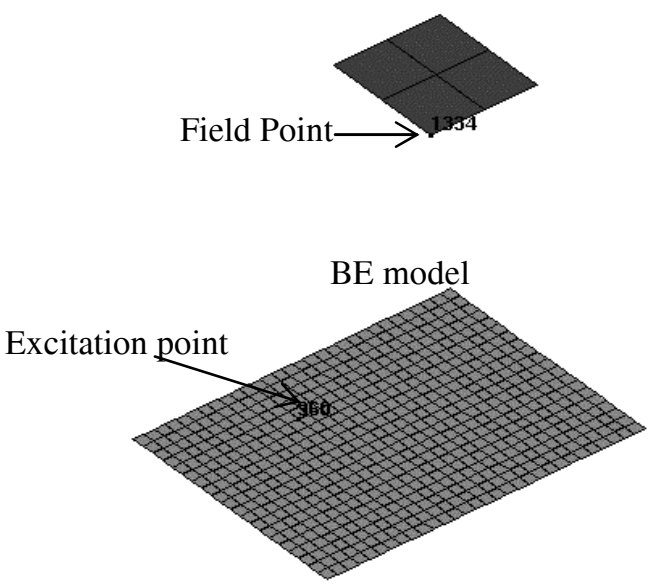

Figure 6. BE plate model

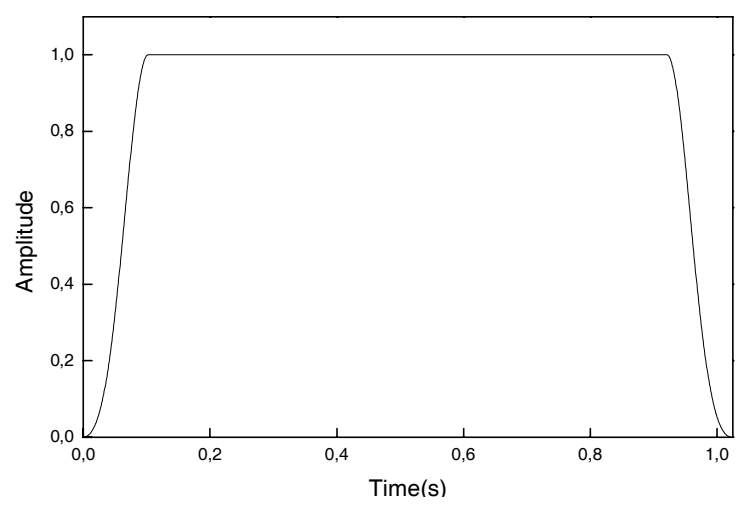

Figure 7. Window for FFT

The BEM results as presented in this paper, are compared in this case to Rayleigh method. This method is based on the hypothesis that each element of the vibrating surface is mounted on an infinite rigid baffle and vibrates independently from the other elements constituting the vibrating surface (Herrin et al., 2003). Consequently, the total pressure field is obtained by summing the pressure generating by each element. Compared to BEM, this one is very fast since it does not need to construct and to solve a linear system. In fact, for this problem, BEM takes $30 \mathrm{mn}$ to construct and to solve the linear system whereas Rayleigh method gives final result in few seconds. Figure 8 show that this method gives a good approximation of the BEM solution. It leads to good results when applied to a 
vibrating flat surface. However, using Rayleigh method for more complex problems can lead to some wrong conclusions and decisions because one can never be sure in which case this method yields to accurate results.

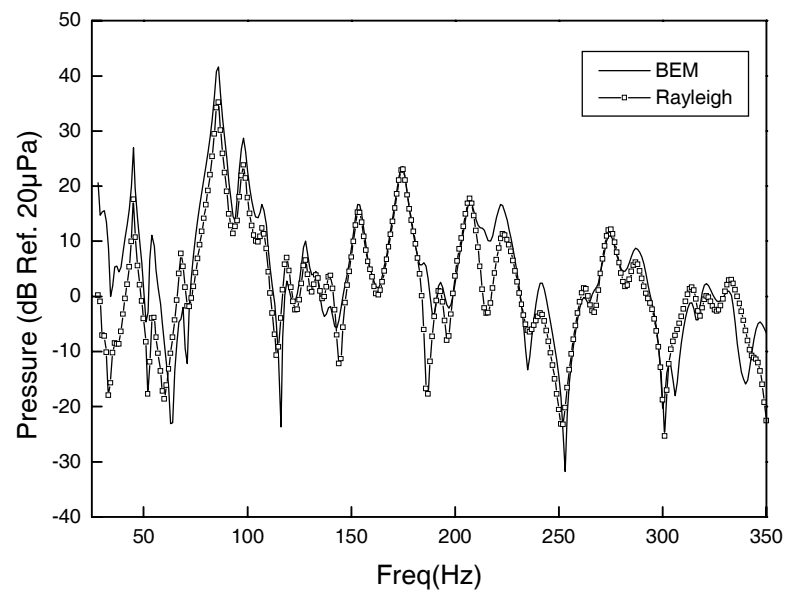

Figure 8. Pressure at the field point: comparison between BEM and Rayleigh method

\section{Conclusion}

In this paper, the acoustic pressure has been calculated by using the structure velocity, obtained from a finite element code, as boundary condition for BEM. The FFT allows to transform these temporal velocities into frequency domain. In order to preserve the periodicity of the velocity signals, a window is employed instead of the rectangular one. The BE code has been checked for an acoustic problem. In addition, the presented structure-acoustic interaction simulation has been applied to a vibroacoustic problem and compared to Rayleigh method results.

The method as presented in this paper is not applicable to the strong coupling problems. It still limited only for air acoustic problems.

\section{Acknowledgements}

The authors thank S. Schneider and S. Marburg for providing GMRES solver. 


\section{References}

Barrett R., "Templates for the solution of linear systems: building blocks for iterative methods", SIAM, Philadelphia, 1994.

Estorff O. V., Boundary elements in acoustics: advance and applications, WITTPRESS, Boston, 2000.

Herrin D. W., Martinus F., Wu T. W., Seybert A. F., "A new look at the high frequency boundary element and Rayleigh integral approximations", Soc. Auto. Eng. Inc., 03NVC114, 2003.

Marburg S., Schneider S., "Performance of iterative solvers for acoustic problems. Part I. Solvers and effect of diagonal preconditionning. Engineering analysis with Boundary elements", vol. 27, 2003, p. 727-750.

Marburg S., "Developments in structural-acoustic optimisation for passive noise control", vol. $9, \mathrm{n}^{\circ} 4,2002$, p. $291-370$.

Ohayon R., Soize C., Structural acoustics and vibration, London, Academic press, 1998.

Sandberg G., "A new strategy for solving fluid-structure problems", International Journal for Numerical Methods in Engineering, vol. 38, 1995, p. 357-370.

Tournour M., Atalla N., "Pseudostatic corrections for the forced vibroacoustic response of a structure cavity system", Journal of Acoustical Society of America, vol. 107, 2000, p. 2379-2386.

Wu T.W., Boundary element acoustics: Fundamentals and computer codes. Advances in boundary elements, Southampton, Boston, Witpress, 2000. 